vection are mostly restricted to minor Archaean problems. On this basis, the book tends to be overly concerned with the end-products of mantle processes and virtually ignores the mechanisms by which they are derived, and therefore why their operation may have changed with time. Some discussion of crust is also required and the way in which these differences have evolved should have been discussed. Reducing some of the side-lines in the second half and really considering the changing mechanism of tectonic change would then have been more consistent with the title.

The production is excellent and the illustrations are ample; in fact almost too many, as several could have been usefully combined. This tends to apply also to the text, where Windley's desire to give other people's views possibly the vertical variation in the continental

would have been better presented as a clear statement with references. These fcatures tend to make some parts seem rather too much like lecture notes, with slides of published work, than is desirable. Such comments are, however, intended sympathetically, as it would be hard to improve the text without grossly expanding the total length. Nonetheless, although improvements are possible, particularly in considering the mechanism of tectonic change, this book can be unreservedly recommended to final-year and postgraduate students both as a textbook and a source of references for further studies. It must be an essential on any final-year earth science reading list.

D. H. Tarling is Senior Lecturer in the Department of Geophysics and Planetary Physics at the University of Newcastleupon-Tyne, UK.

\section{Dynamic systems}

Synergetics: An Introduction. NonEquilibrium Phase Transitions and SelfOrganisation in Physics, Chemistry and Biology. By H. Haken. Pp. xiit 325. (Springer: Berlin and New York, 1977.) DM72; $\$ 31.70$.

For some time now, it has been the main concern of the author to popularise the subject he has chosen to call "synergetics". To most readers, however, this term is less familiar than the subject itself-the theory of phase transitions in co-operative dynamic systems, applied to examples ranging from physics to sociology. Part of the material presented in this book is already contained in several review articles and conference proceedings, but now $H$. Haken presents in this volume a detailed and comprehensive account of the basic principles and applications of this "interdisciplinary field of research".

The term "synergetics" was prompted by the variety of dynamic systems stemming from physics, chemistry, biology and sociology which show striking similarities at their points of instability. As the book is meant to be a student textbook, the author provides in the first few chapters the mathematical prerequisites. After some introductory sections on probability and information theory, the reader becomes acquainted with stochastic processes (Brownian motion, random walks and their description by Master equations) and deterministic motion (here, the concepts of stability, critical points, hard and soft mode excitations, limit cycles, and so on, are introduced).
The connection between deterministic and stochastic processes (or chance and necessity) is provided by the Fokker-Planck equation approach, which is discussed extensively and used to introduce the Landau theory of phase transitions. Combining all these concepts, the author then explains in a rather formal and abstract way the generation of order by self-organisation. Then, separately, these formal considerations are applied to various examples, Starting from physics (laser and hydrodynamic instabilities), the reader is lead to nonlinear chemical reactions (with their showpieces, Brusselator and Oregonator) and biological systems (models for ecology, evolution and morphogenesis); and, finally, a model for interacting social groups is presented.

This book certainly. serves its purpose as an introductory student text, as it requires little previous knowledge. Besides tradition (synergetics is not part of a usual university curriculum), the only obstacle to its wide distribution among students may be its fairly high price

Experienced readers will certainly profit from the variety of examples presented and the rather detailed reference section, a good guide to the topical literature. It is a pity, however, that some of the important work of several Japanese groups on nonlinear chemical reactions is missing-instead, the author has chosen to cite unpublished work carried out by his own group. But this minor weakness is by far outweighed by many advantages.

R. Schranner

R. Schranner is a Postdoctoral Fellow of the Max-Planck Institute of Biophysical Chemistry, Göttingen, FRG.

\section{Quantum mechanics}

The Uncertainty Principle and the Foundations of Quantum Mechanics. Edited by W. C. Price and S, S. Chissick. Pp. 572. (Wiley-Interscience: London and New York, 1977.) £19.50; \$38.

BEING a collection of articles, this book cannot be quite faithful to its title: particularly since it is just one book in a series of books, each designed as a tribute to one of the founders of modern physics. Clearly, such publications are not intended to provide anything like a systematic and critical survey of a subject, especially when the latter is highly structured, as is the case with regard to the foundations of a discipline. But what such books can do is to present significant partial results and to direct the reader's attention toward recent specialised research. This is in fact what is achieved, and in a highly satisfactory way, by this book.

Although I benefited from several of the valuable articles the book contains, let me follow my own taste in selecting just one for review: the contribution by G. Lanz. This paper bears on what is often considered to be the only problem remaining in the foundations of quantum mechanics, namely the problem of measurement. Its description of the difficulties besetting that problem is clear. So is its classification of the two conceptions of quantum mechanics that (barring non-local hidden variables) one may still discuss quantum mechanics as the basic theory of physics or quantum mechanics as a mere theory of microsystems, anchored on a (still to be created) theory of macrosystems. The author considers that the first view should be dismissed because of insuperable difficulties with the problem of measurement. As for the second conception, it obviously requires a theory of macrosystems, and an unambiguous definition of what a macrosystem is.

Lanz acknowledges that he has until now no completely definite answers to such questions. He sketches, however (with some profusion of mathematical detail), an approach based on Ludwig's formalism which he believes is promising. It involves a limiting procedure the physical meaning of which is unfor tunately not discussed. The general idea is to extract from the $\mathrm{N}$-body theory a part that could be used for a theory of macrosystems; the rest could then be thrown away as physically irrelevant. An interesting but perhaps premature question is whether the future theory will be able to define macrosystems in such a way that at least some finite (and really existing) systems are macroscopic.

Bernard d'Espagnat

Bernard d'Espagnat is Professor of Theoretical Physics at the University of Paris-Orsay. 